\title{
Modelling of Indoor Lighting Conditions in Buildings for Control Design Purposes
}

\author{
Vito Logar ${ }^{1 *}$ \\ ${ }^{1}$ Faculty of Electrical Engineering, University of Ljubljana, Tržaška 25, 1000 Ljubljana, Slovenia; \\ *vito.logar@fe.uni-lj.si
}

Simulation Notes Europe SNE 26(4), 2016, 267 - 276

DOI: $10.11128 /$ sne. $26 . t n .10358$

Received: November 25, 2016

Accepted: December 5, 2016 (Special Issue Review)

\begin{abstract}
Lighting conditions in buildings and efficient use of solar energy are a subject of considerate attention in order to provide sufficient living comfort and to reduce the energy use. For this reason numerous methods and techniques, practical and theoretical, have been developed. In this paper a theoretical approach to modelling of the indoor lighting conditions is proposed, based on fuzzy black-box modelling. The presented model is able to estimate indoor illuminance levels as its outputs, by using measured external conditions as its inputs. The model can be used to study the influence of both controllable and uncontrollable variables to the indoor lighting conditions, such as weather, time of the year, blinds position, electric lighting and others. Furthermore, using the above model studies on control design can be performed in order to obtain algorithms for maximal use of the solar energy and to minimize the energy consumption. The study has shown that a fuzzy illuminance model can estimate the indoor illuminance levels comparable to the measured data. Small error measures show that similar modelling approach can be used in order to integrate the proposed model into other environments and can further be used for simulations on indoor lighting comfort, control design or modelbased control.
\end{abstract}

\section{Introduction}

Indoor lighting conditions and the efficient use of solar energy have become very important in recent decades, both in terms of overall living comfort $[1,2]$ and energy-efficient buildings $[3,4,5,6]$.
A summary of the research carried out to date in the area of buildings' energy efficiency, buildings' energy performance and buildings' processes modelling can be found in the excellent review paper by Foucquier et al. [7]. Furthermore, sufficient daylight conditions have been proven to have a beneficial effect on human health [8]. Numerous approaches to controlling indoorilluminance conditions have been proposed, most of which attempt to either achieve constant indoorilluminance levels, so as to provide sufficient living comfort, or to maximize the use of solar energy, while still providing acceptable lighting conditions $[9,10,11$, $12,13]$. Together with the modelling of light flux, indoor light intensities and surface illuminances, which usually represent the basis for control design techniques, have also been the subject of much attention. Furthermore, many methods exist that are able to provide approximate illuminance-level prediction in a certain position in a room, given its geometry, global orientation, the position of the sun, the surface characteristics and/or the weather conditions/measurements $[14,15$, 16]. Moreover, a study performed by Lindelöf [17] proposes a fast daylight model, able to obtain indoor illuminances as a linear combination of the external global and diffuse radiations, validated using the Radiance model, which can be used as a replacement for the real system of embedded controllers. Similarly, available software tools, i.e., Radiance, Daysim, Skyvision $([18,19,20])$ and many others, are also able to calculate more-or-less accurate illuminance levels for a given position in a room; however, significant knowledge of the modelled system (complete geometric and photometric characteristics of the room, inventory, windows, blinds, lights, etc.) and the software itself are needed, in order to ensure accurate results. A lot of the existing approaches rely on known mathematical daylighting concepts and thus try to describe the physical relations between the input and output variables. 
If the measurements of the real environmental conditions are available, a black-box approach to the calculation of the indoor illuminance can be introduced as one of the modelling possibilities. Black-box models have proved to be a useful tool for the modelling of processes whose characteristics, relations and dynamics are not exactly known or are harder to model with conventional approaches.

The objective of this study is to propose a black-box approach to indoor-illuminance estimation by using a fuzzy inference model. The proposed methodology results in the development of a model that describes the relations between its inputs: horizontal unobstructed illuminance (external illuminance), global and diffuse solar radiations, the positions of the blinds and the status of the lights; and its output: the estimated indoor illuminance. The method is, from the input/output point of view, similar to some existing methods, i.e., Lindelöf [17]; however, the methodological approach between the proposed and the existing methods is entirely different.

One of the main advantages of the proposed method is the simple design and parameterisation of the model, which does not require any knowledge about the modelled system, since the model's parameters, which define the input/output relations, inherit the room's characteristics, implicitly defined in the obtained measurements. Meaning that the room's characteristics, such as: geometry, indoor surfaces' reflectances, blinds' reflectances, quantity, sizes and positions of the windows, lights and furniture; and also the position of the indoor illuminance sensor, reflect in the measured indoor illuminance. Moreover, a change in either the room characteristics or the position of the sensor, if sufficiently large, also affects the measured value. After the model is parameterized (trained) and validated using the particular input/output measurements of interest, simulated or otherwise acquired input data can be used, replacing the actual measurements. The inputs defining the blinds' positions and the lights' status either need to be predetermined, adjusted manually or by means of the controller. Since the method uses measurements instead of physical characteristics in order to define the relations of the model, programming skills and the effort to manually design the room interior are not needed.

The proposed structure of the fuzzy models is very simple (5 inputs, 1 output, 3 Gaussian membership functions per input and 3 fuzzy rules) and the fuzzification/defuzzification procedures are simple vector multiplications.
The simplicity of the model is reflected in the fact that it is a fast model, with short evaluation times, which facilitates its inclusion in other applications or control algorithms. Finally, even though the model's structure is simple, the validation results have very accurate estimations in comparison with the measurements.

Although the proposed methodology has advantages, the fuzzy approach also has some drawbacks, which need to be considered when adopting the concept. In contrast to methods based on the physical modelling of the daylighting processes and pre-programmed algorithms, which normally require only the input part of the data in order to obtain the output, the fuzzy approach requires both the input and the output part of the data in order to parameterize the model using an automated training procedure. After the model is parameterized, only the input data is required. Moreover, since the model is based on measured data, which defines the room's characteristics, the calculation of the indoor illuminance under different conditions (e.g., different geometry, reflectances, sensor positions, etc.) needs a re-training of the model with new measurements. From this point of view, other tools like Radiance outperform the proposed method, since they are able to calculate a more-or-less accurate indoor illuminance for an arbitrarily positioned surface $[23,24]$.

The purpose of the study is not to propose a specific model that would represent a general solution for all possible situations (like Radiance, for instance), but to propose a simple methodology for how to obtain a model for a particular environment, where the model is characterized as fast, accurate and easy to obtain, without excessive knowledge of the particular problem. Furthermore, since the in-depth studies on, e.g., building automation, control design, energy conservation, living comfort, etc., are practically impossible to perform on real systems, due to varying weather conditions and poor repeatability, the use of a relatively simple illuminance model of sufficient accuracy in combination with the simulation procedures allows fast and repeatable testing of the designed algorithms or the model-based control of real processes.

\section{Indoor Environment}

The following section gives a description of the indoor environment, whose measurements are used as a basis for the fuzzy black-box model's development and the parameterisation. 
The indoor environment consists of a room with dimensions of $7.49 \mathrm{~m} \mathrm{x} 4.93 \mathrm{~m} \mathrm{x} 3.88 \mathrm{~m}$ ( $1 \times \mathrm{w} \times \mathrm{h})$, a floor area of $38.80 \mathrm{~m}^{2}$ and a volume of $163.40 \mathrm{~m}^{3}$, with one outside wall that has a window, facing south-west (rotated approximately $30^{\circ}$ counter clockwise from the east-west direction), where the outside wall is the longest wall. The room is located on the $4^{\text {th }}$ of 5 floors in a building with no external obstacles that would obscure the light flow through the window (lat: 46.045737, lon: 14.494851). The area of the window is $11.4 \mathrm{~m}^{2}$, with installed venetian blinds. The transmission of visible light through the window is $80 \%$. The room characteristics in terms of the photometry are the following: grey floor (35\% reflectance), white ceiling ( $80 \%$ reflectance), white walls and beige furniture (average $65 \%$ reflectance). Figure 1 shows the floor plan of the particular room, with the marked positions of the sensors (indoor and external illuminance, global and diffuse solar radiation - placed on the roof of the building, blinds' position), the window and the blinds.

The studied indoor environment is equipped with an automation, supervisory control and data-acquisition system (SCADA), which is composed of three distinct parts: the sensor array, the process and supervision level, and the data-acquisition level. The system measures the necessary values, such as the global and diffuse solar radiation, the external illuminance, the position of the blinds (and other values not relevant to this study) and controls the indoor-illuminance levels (and other values not relevant to this study) using the motorized venetian blinds and the electric lighting. The sensor for external illuminance (Thermokon LI65 outdoor light sensor) is mounted vertically on the facade beside the window and is capable of measuring the illuminance in the range from 0 to 20,000 lux. The sensors for the global and diffuse solar radiation (Kipp \& Zonen CM7B pyranometer and albedometer) are mounted 2 floors higher, horizontally on the roof of the building and are measuring the solar radiation in the range 305 to $1,800 \mathrm{~nm}$ from 0 to $1,400 \mathrm{~W} / \mathrm{m}^{2}$. The indoor illuminance sensor (Thermokon LI04) is placed horizontally on the workbench/desk (at a height of approximately $0.9 \mathrm{~m}$ ) and is capable of measuring the illuminance in the range from 0 to 2,000 lux. A complete description of the automation system and the applied control algorithms can be obtained from the paper by Košir et al. [13].

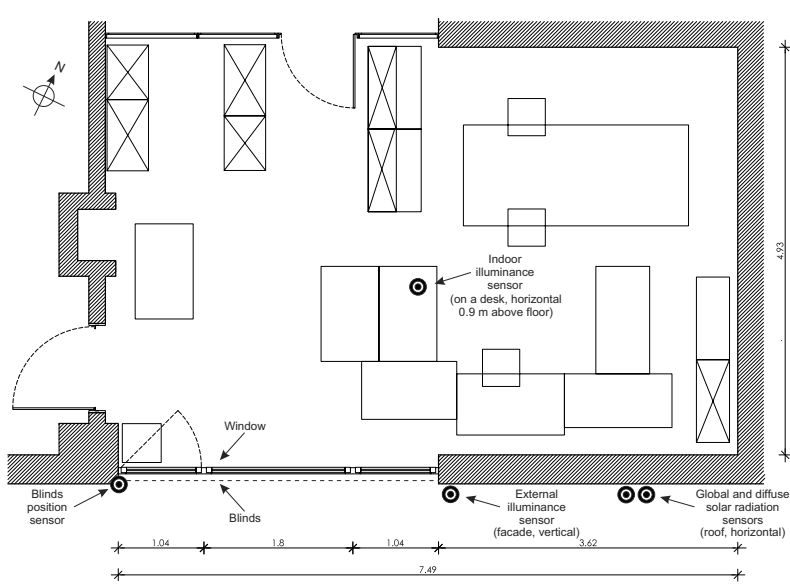

Figure 1: Floor plan of the modelled room, with the marked positions of the sensors (indoor and external illuminance, global and diffuse solar radiation (placed on the roof of the building), blinds' position), the window area and the blinds.

The global and diffuse solar radiation are measured in $\mathrm{W} / \mathrm{m}^{2}$, the external illuminance in lux, while the blinds' position can take values between 1 and $5\left(1-0^{\circ}\right.$ slat angle (vertical), 2 - $30^{\circ}$ slat angle, 3 - $60^{\circ}$ slat angle, 4 $90^{\circ}$ slat angle (horizontal) or 5 - blinds completely retracted) and the lights' status can be either 0 (OFF) or 1 $(\mathrm{ON})$.

\section{Fuzzy Model}

The concept of the black-box theory relies on understanding something entirely in terms of its function, without knowing the background or the mechanisms that enable this functionality. From this point of view, machine-learning techniques, among which are also fuzzy-inference systems, as one of the black-box approaches, can be considered as a mechanism of this black box for the input-to-output mapping of the data space. Meaning, if an appropriate structure of the system is designed, an arbitrary nonlinear function between the system's inputs and outputs can be described by the fuzzy mechanism. Since the black-box approach has no physical background to the particular process, the function describing the input-to-output space can be as close to the real physical relation as the learning data can describe it. Such a system can, therefore, be definitely valid only in the vicinity of the mapped data space. 
This could be considered as one of the drawbacks of the black-box approach, as if the learning-data inputoutput relation is missing, the later incidence of such an input could lead, but not necessarily, to incorrect output estimations and should be experimentally validated. However, if sufficient data is available, the black-box approach has certain advantages over conventional modelling approaches. As mentioned before, when the structure of the black box is specified, its relations are determined only according to the input and output data, which eases the modelling for those who are, or are not, familiar with the process. Physical relations, process characteristics and other properties that could be hard to describe mathematically are replaced by the proper selection of the fuzzy structure (which can be obtained experimentally) and only limited knowledge (usually the order of the process) of the physical process is needed to build the process model. In this manner, the proper structure and parameterisation (pre-programmed automated learning procedures) of the fuzzy mechanism ensures that the model's parameters, which define the input/output relations, inherit the room's characteristics, implicitly defined in the obtained measurements.

\subsection{Structure of the model}

The proposed black-box illuminance model is based on a Takagi-Sugeno (TS) fuzzy-inference system (FIS) [25] that was trained using real environment measurements as the inputs and outputs. The model, in TS form, approximates a nonlinear system by smoothly interpolating affine local models [25]. Each local model contributes to the global model in a fuzzy subset of the space characterized by a membership function. The affine TS model can be used to approximate any arbitrary function with any desired degree of accuracy [26, $27,28]$. The generality can be proven with the StoneWeierstrass theorem [29], which suggests that any continuous function can be approximated by a fuzzy-basis function expansion [30].

Since the light-flow dynamics can be, from the room-illuminance point of view, considered as infinitely fast or instantaneous, the black-box model is regarded as a static model from the modelling perspective. This means that a change in either the global or the diffuse light, the blinds' position or the lights' status has an immediate effect on the change in the observed illuminance, with no transitional dynamics, meaning that the value of the current illuminance is completely independent of the previous illuminance sample.
The fuzzy model uses the external illuminance multiplied by the position of the blinds, the global and diffuse solar radiation, the lights' status and the blinds' position as the inputs and the indoor illuminance as the output. The reason for using the multiplied external illuminance and blinds position as the input is that better estimation results can be achieved, in comparison to the results with only external illuminance as the input, which is a consequence of a prior virtual increase in the external illumination (multiplied by a factor from 1 to 5), dependent on the position of the blinds. The multiplication, therefore, implies that more light is entering the office when the position of the blinds is higher (higher slat angle) or if the blinds are completely retracted.

This implication allows better estimations of the indoor illuminance by the fuzzy model, since the particular input already partially describes the relation between the external illuminance and the blinds' position. However, the relation between the external illuminance, the blinds position and the indoor illuminance is not linear, as implied by the multiplication, but nonlinear, which is later defined by the corresponding fuzzy membership functions and the antecedent rules. The use of the global and diffuse radiation as two of the inputs (besides the external illuminance, the blinds' position and the lights' status) is optional and leads to better estimation results, in comparison with the results that have the global and diffuse radiation measurements omitted.

The reason for using the blinds' position as one of the inputs, despite the fact that it has already been used in the multiplication with external illuminance, is that even better estimation results can be achieved in comparison with the results that have this input omitted, which could be a consequence of the nonlinearity of the process caused by the blinds' reflectance, which is thus represented more accurately. Figure 2 shows a schematic representation of the model.

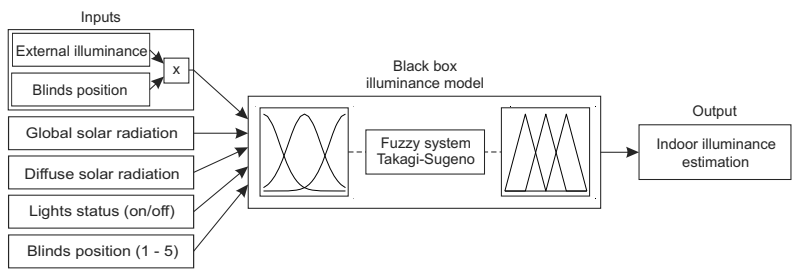

Figure 2: Schematic representation of the fuzzy black-box illuminance model. 


\subsection{Illuminance reconstruction}

This section explains an optional method for simple external illuminance reconstruction, which is necessary when the illuminance sensor has a limit (in this case 20,000 lux). If sensors with higher limits (100,000 lux or higher) are used to obtain the measurements, the external illuminance reconstruction is not needed and the measured external illuminance can be used as an input to the fuzzy model directly.

Knowing that during sunny summer days the external illuminance can go above 100,000 lux, the missing (saturated) data needs to be reconstructed in order to achieve better estimations of the calculated value. As the fuzzy model also uses the global and diffuse solar radiations (among others) as its inputs, it is able to sufficiently well predict the indoor illuminance levels, even when the external illuminance goes above the sensor's limit (20,000 lux) and its value is not reconstructed; however, estimations during peak daylight are less accurate. The reconstructed signal is far from being equal to the actual illuminance levels; however, according to the validation results presented, an approximate estimation of the external illuminance suffices. Since the daily illuminance trend follows approximately the same shape, it can be approximately reconstructed according to the total saturation time of the external illuminance sensor, as given in the equation 1 :

$t_{\text {sat }}=t_{\text {end }}-t_{\text {start }}$

$q_{\text {reconstruct }}\left(t_{\text {start }}: t_{\text {end }}\right)$

$=1.5$

$\times 10^{4} \sqrt{\frac{\left(-\left(\text { linspace }\left(-t_{\text {sat }}, t_{\text {sat }}\right)\right)^{2}+t_{\text {sat }}^{2}\right)\left(\text { linspace }\left(-t_{\text {sat }}, t_{\text {sat }}\right)+t_{\text {sat }}\right)}{2 t_{\text {sat }}}}$

$$
q_{i l l}=q_{i l l}+q_{\text {reconstructed }}
$$

where $t_{\text {start }}$ and $t_{\text {end }}$ represent the beginning and the end of the sensor saturation in hours, $t_{s a t}$ represents the overall length of the saturation in hours, $q_{\text {reconstruct }}$ represents the reconstructed illuminance signal to be added to the measured illuminance signal in lux, $q_{\text {ill }}$ represents the external illuminance signal in lux and the command linspace $(a, b)$ represents a vector of linearly spaced values between $a$ and $b$.

Figure 3 shows the comparison between the measured and the reconstructed external illuminance, as obtained by equation 1 for a part of the training data ( 5 days in early August), where the sensor reaches its upper limit.

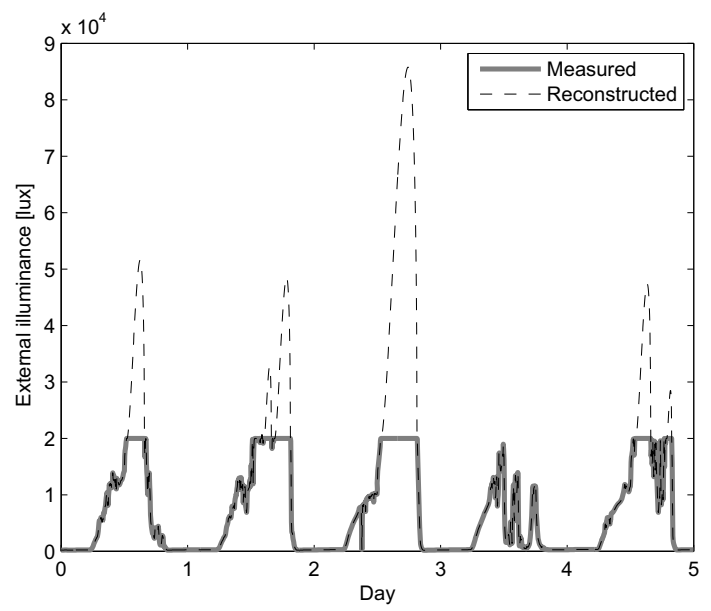

Figure 3: Comparison between the measured and the reconstructed external illuminance for 5 days in early August.

As can be seen in figure 3 the measured external illuminance is saturated at 20,000 lux for several hours around midday, while the reconstructed illuminance reaches up to almost 90,000 lux, which seems reasonable for sunny summer days. Equation 1 and the corresponding results are obtained experimentally, without any physical background and are not validated nor taken from any other source, but merely a rough estimation of what the external illuminance should be like. If the illuminance sensor has a limit that is high enough, such an estimation is not needed.

\subsection{Parameterisation of the model}

The parameterisation of the fuzzy model, also known as the training, was carried out for 1 month of different measurements with a sample time of $15 \mathrm{~s}$. The data was chosen in a manner that covered all four seasons and as many real-world situations as possible, i.e., sunny, cloudy, foggy weather, different sun azimuths and elevations, quick illuminance changes due to partial cloudiness or incoming thunderstorms, operation of lights, shading, etc. Shorter or longer periods of measurement data can be used to train the model; however, using less training data results in a less versatile and robust model, which is sufficiently accurate only for similar conditions. On the other hand, using more training data results in a more versatile and robust model; however, extended data only increases the model's performance by a smaller amount. The fuzzy model uses three data clusters for each input and the Gaussian membership functions. 
An adaptive, neuro-fuzzy, hybrid learning algorithm (ANFIS), as implemented in the Matlab environment, was used for the model training.

Figure 4 shows a part (5 days in early August) of the input data (otherwise 30 days) that was used for the model training, including the external illuminance, the global and diffuse solar radiation, the blinds' position and the lights' status.
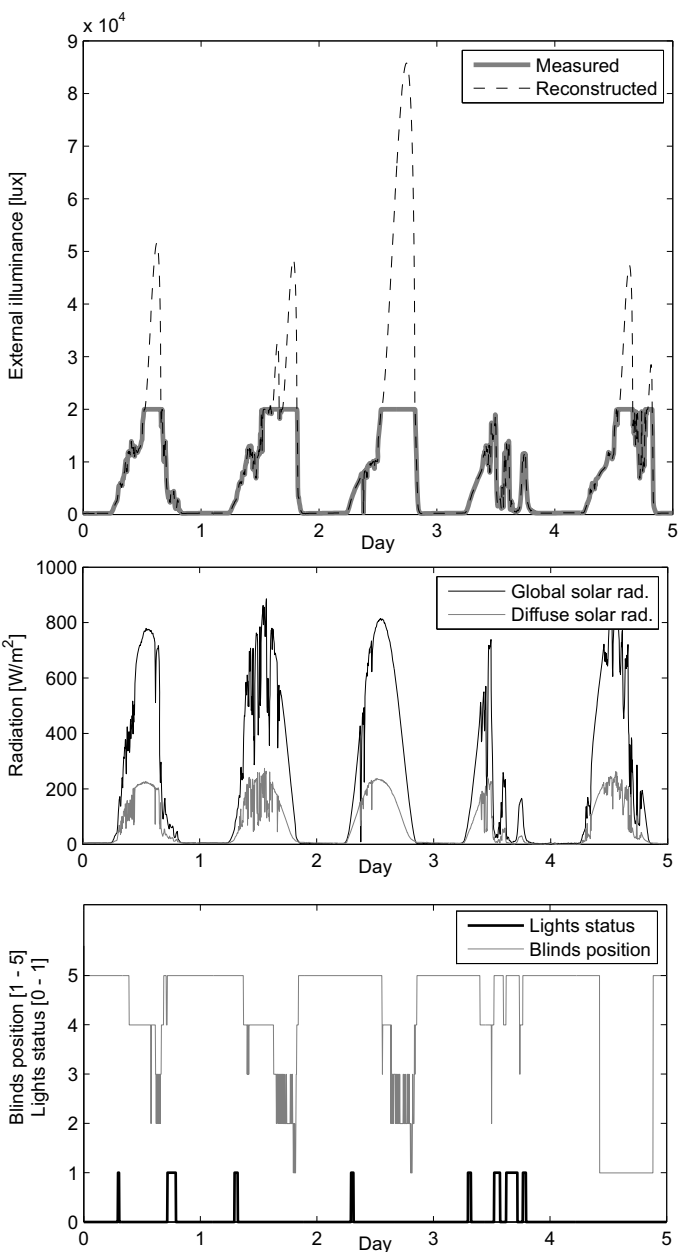

Figure 4: A part (5 days in early August) of the input data for the model training, including the reconstructed external illuminance (upper panel), the global and diffuse solar radiation (middle panel), the lights' status and the blinds' position (lower panel).

As is clear from figure 4, for the selected days, the global and diffuse radiation take values between 0 and $900 \mathrm{~W} / \mathrm{m}^{2}$, the external illuminance between 0 and more than 20,000 lux (reconstructed above 20,000 lux as shown in figure 3 ), the blinds' position can be in the range from 1 to 5 and the lights' status can be either 0 or 1 , as already described.

\section{Results}

Using real measurements, the model was trained using an ANFIS training algorithm. Figure 5 shows the shape and distribution of the trained-model membership functions for each input. The membership functions, which characterize the input-output space, define the effect of a given input value on the computed output value. Since the presented model has five different inputs and three data clusters, each input has three membership functions with different centres and kurtosis.
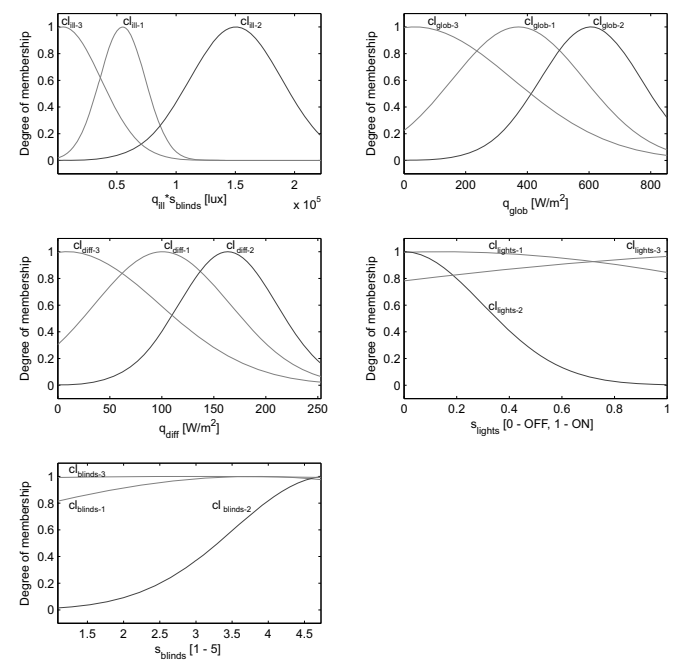

Figure 5: Shape and distribution of the fuzzy membership functions on all five inputs for all three data clusters as obtained by the ANFIS training algorithm.

Figure 5 shows the shape and the distribution of the membership functions, which contribute to the value of the output, i.e., the indoor illuminance level, which is obtained using the following equations:

$$
\begin{aligned}
\text { out }_{1}= & {\left[q_{\text {ill }} s_{\text {blinds }} c l_{\text {ill }-1}, q_{\text {glob }} c l_{\text {glob-1 }}, q_{\text {dif }} c l_{\text {dif }-1},\right.} \\
& \left.s_{\text {lights }} c l_{\text {lights }-1}, s_{\text {blinds }} c l_{\text {blinds }-1}\right] \times \\
& {[0.0002238,-0.2234,2.091,453.3,182.4]^{T}-} \\
& 373.1 \\
\text { out }_{2}= & {\left[q_{\text {ill }} s_{\text {blinds }} c l_{\text {ill }-2}, q_{\text {glob }} c l_{\text {glob }-2}, q_{\text {dif }} c l_{\text {dif }-2},\right.} \\
& \left.s_{\text {lights }} c l_{\text {lights }-2}, s_{\text {blinds }} c l_{\text {blinds }-2}\right] \times \\
& {[0.002637,0.3512,1.361,-99.01,-16.13]^{T}-} \\
& 168.1 \\
\text { out }_{3}= & {\left[q_{\text {ill }} s_{\text {blinds }} c l_{\text {ill }-3}, q_{\text {glob }} c l_{\text {glob-3 }-3}, q_{\text {dif }} c l_{\text {dif }-3},\right.} \\
& \left.s_{\text {lights }} c l_{\text {lights }-3}, s_{\text {blinds }} c l_{\text {blind }-3}\right] \times \\
& {[0.01307,-0.8077,1.146,128.5,-1.442]^{T}+} \\
& 19.17
\end{aligned}
$$


where $q_{\text {ill }}$ represents the external illuminance, $s_{\text {blinds }}$ represents the blinds' position, $q_{g l o b}$ and $q_{d i f}$ represents the global and diffuse solar radiation, $s_{\text {lights }}$ represents the lights' status and $c l_{x-n}(x$ - correspondent input, $n$ cluster number) represents the degree of membership (from 0 to 1 ) of the given input to the corresponding cluster. Finally, the indoor illuminance $q_{\text {in }}$ is obtained using the following equation:

$$
q_{\text {in }}=q_{\text {out } 1}+q_{\text {out } 2}+q_{\text {out } 3}
$$

The membership functions shown in figure 5 and the fuzzy rules described by equations 2 to 5 define the socalled mapping of the input-output data space. Generally, each input contributes to the combined output according to the corresponding membership functions, its centre position and kurtosis and the equations 2 to 5 .

Figure 6 shows the validation of the model by comparing the measured and model-estimated indoor illuminances for 12 different days (days 1-3: June, days 46: September, days 7-9: December, days 10-12: March), covering different solar positions and climatic conditions. The model was validated using the data from different days than were used for the model training.

As can be seen in the first graph of figure 6 the indoor illuminance levels obtained with the fuzzy model in general correspond to the measured illuminance levels. The indoor illuminance measurements during the periods of daylight are approximately limited to a range from 400 to 1,600 lux and are a consequence of the external illuminance conditions, the position of the blinds and the status of the lights. Lower indoorilluminance values occur during the morning and early afternoon, while these values are higher later in the day, which is a direct consequence of the room's orientation (direct sunlight to the window area appears in the late afternoon, around $6 \mathrm{pm}$ ). The illuminances at night, both measured and estimated, are around 10 lux, and not close to 0 lux as expected, which could be a consequence of the skyglow (light pollution) and the neighbouring street lighting.

The second graph of figure 6 shows that external illumination measurements reach over 20,000 lux (near 80,000 lux estimated) in the first 6 validation days' data (June and September), which is reasonable for this period of the year. However, shorter intervals of sensor saturation appear in days 4 to 6 (September), due to the shorter intervals of daylight, which is also reflected in lower estimated peak illuminances (up to 40,000 lux).
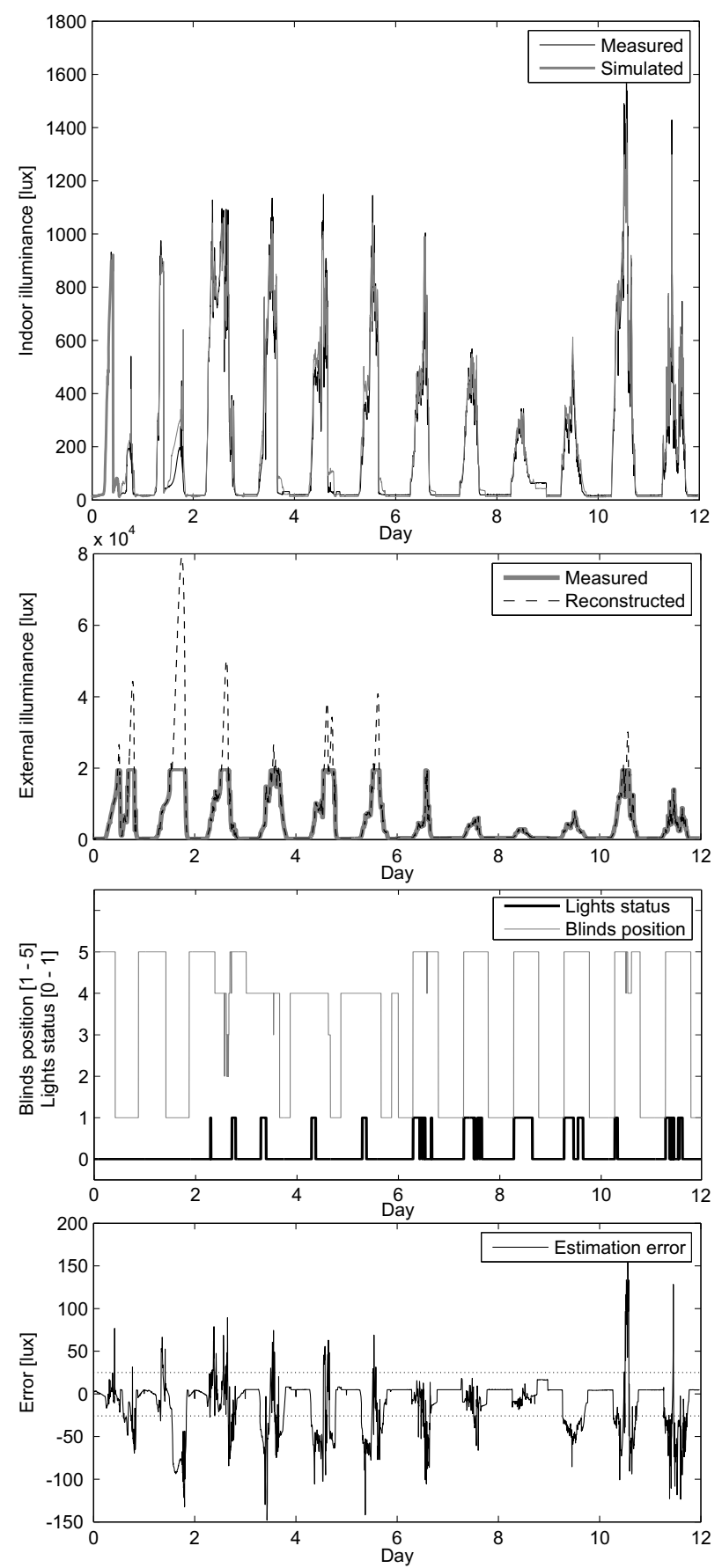

Figure 6: Illuminance model validation; first graph shows the comparison of the measured (black line) and model simulated (grey line) indoor illuminances for 12 different days (days 1-3: June, days 4-6: September, days 7-9: December, days 10-12: March); second graph shows the measured external illuminances with reconstruction; third graph shows the position of the blinds and the lights' status, respectively; fourth graph shows the illuminance estimation error (dashed vertical lines show the mean absolute error interval). 
Furthermore, individual occurrences of illuminance below 20,000 lux suggest partial cloudiness or incoming thunderstorms (i.e., day 1). The last 6 validation days' data (December and March) mainly exhibit illuminances below 20,000 lux, typical for the winter and spring seasons. Days 6, 11 and 12 suggest sunny weather with partial cloudiness (day 12), while days 8 to 10 suggest a full overcast or all-day fog, which is typical for this time of the year.

The fourth graph of figure 6 shows the error between the measured and the model-predicted illuminances. Individual error spikes between the measured and the simulated data, visible at days $1-6,11$ and 12 , reaching up to approximately 150 lux can be observed. The spikes mostly occur at peak daylight, when the external illuminance is the highest, which implies that significant deviations between the actual and the reconstructed illuminances occur. Namely, the reconstructed illuminance is a smooth curve, neglecting the occurrence of individual clouds, which rapidly decrease the measured illuminances and cause fast illuminance fluctuations.

The problem is not a flaw of the presented method, but occurs solely due to the external illuminance sensor limit and its reconstruction and can easily be avoided using an illuminance sensor with a limit above 100,000 lux, which allows proper measurements of the illuminances above 20,000 lux. On average, the error between the measured and the estimated illuminances (mean absolute error - MAE) is approximately 25 lux (dashed vertical lines), which can be considered as a good model approximation of the real data. Furthermore, the rootmean-square error (RMSE) and the mean-bias error (MBE), as two of the measures of estimation reliability, are $12.60 \%$ and $7.76 \%$, respectively, and suggest the satisfactory reliability of the fuzzy estimator (RMSE lower than $35 \%$ and MBE lower than $15 \%$ ) [23].

Based on the presented comparison between the measured and the estimated indoor illuminances and the error measures (MAE, RMSE and MBE), the validation of the model can be considered as successful. The study has shown that the presented fuzzy black-box approach can be used as a satisfactory approximator for the indoor illuminance conditions in buildings.

\section{Conclusion}

The paper presents a fuzzy black-box modelling approach to estimating the illuminance of an indoor environment. The model is used to estimate the illuminance level at a certain point on a surface, given the required input values, and is easy to design and implement. The method uses real environment measurements, such as external illuminance, global and diffuse solar radiations, blinds' position, lights' status and indoor illuminance, in order to define the relations and obtain the parameters of the model.

After the model is parameterized using the particular input/output measurements of interest, simulated or otherwise acquired input data can be used, replacing the actual measurements if desired. The study has shown that a relatively simple fuzzy structure can be used in order to estimate the indoor illuminance at a particular spot. Since the fuzzy approach is based on simple mathematics and the model's structure only incorporates three membership functions per input and three fuzzy rules, the estimation of the indoor illuminance is very fast, allowing the use of the model in broader online or offline environments. Furthermore, low estimation errors $(\mathrm{MAE}=25 \mathrm{lux}, \mathrm{RMSE}=12.60 \%, \mathrm{MBE}=7.76 \%)$ suggest a satisfactory reliability for the proposed estimator.

The fuzzy black-box approach has its advantages and flaws, when compared to other similar methods, which need to be considered when deciding for the appropriate illuminance-estimation method. Briefly, the advantages of such an approach are the ease of model design and parameterisation, an accurate estimation, the mathematical simplicity, a quick calculation and the fact that only limited knowledge of the process is needed. Since the fuzzy model is parameterized from the measured data, the model's structure can also be used at different latitudes, different building orientations, window sizes, indoor photometric and geometric characteristics, etc., but only if the data is available to properly parameterize the model.

This claim has not been experimentally validated; however, following the essence of the black-box approach, i.e., to define a relation between the model's inputs and outputs without extensive knowledge about the background to the problem, but only possessing the required input/output data, the claim is reasonable. 
While the proposed approach has certain advantages over other methods, it also has some general flaws, which mainly originate from the need for measurements. A fair number of measurements, in as many real situations as possible, are needed in order to obtain a robust and versatile model that covers a broad range of climatic conditions (external illuminance, solar radiation) and other influential variables (blinds, lights). Moreover, the proposed approach is able to estimate the illuminances for only one position; thus, if the illuminance levels of different positions in a room are required, measurements from multiple illuminance sensors should be obtained and used to train a multipleoutput fuzzy model.

During the study it was discovered that, depending on the desired model versatility for different conditions, measurements for at least a few days (3-5) are needed in order to parameterize the model to satisfactorily estimate the illuminance for similar inputs. If estimations for a broader range of inputs are needed, at least 15 days of data are required. In order to obtain the results as shown in this paper, 30 days of data for different climatic conditions should be used. The study revealed, that the model performs surprisingly well even if the training data is obtained only during the summer (due to higher external illuminances and solar radiations) and the model is used for estimations in the other three seasons, i.e., training data: 15 consecutive days in July, validation data: 10 consecutive days in January, yields: MAE $=35$ lux, $\mathrm{RMSE}=18.1 \%, \mathrm{MBE}=12.2 \%$. Using a method in this manner, a relatively small amount of modeltraining data is needed, which can be obtained quickly in exchange for a slightly lower accuracy of the model.

The black-box fuzzy model for indoor illuminance estimation, as presented here, is not primarily intended to be used as a stand-alone application, although it can be, but to be included in broader test environments, useful for a variety of studies, for instance: building automation, living comfort, energy conservation, control design, etc. Since such studies are practically impossible to perform on real systems, due to varying weather conditions and almost no repeatability, a combination of different dynamic models (thermal, visual and air quality) and simulation procedures is the most frequently used approach.
Therefore, the use of a simple illuminance model of sufficient accuracy allows quick and repeatable testing of the particular methods. Since the model is reliable and allows for a quick estimation of the illuminance, it can also be used in a similar way to the model presented by Lindelöf [17], i.e., as a model-based control approach using embedded controllers.

\section{References}

[1] Kim JT, Kim G. Overview and new developments in optical daylighting systems for building a healthy indoor environment, Building and Environment 45 (2010) 256269.

[2] Huang L, Zhu Y, Ouyang Q, Cao B. A study on the effects of thermal, luminous, and acoustic environments on indoor environmental comfort in offices, Building and Environment 49 (2012) 304-309.

[3] da Fonseca RW, Didon''\{e\} EL, Pereira FOR, Using artificial neural networks to predict the impact of daylighting on building final electric energy requirements, Energy and Buildings 61 (2013) 31-38.

[4] da Silva PC, Leal V, Andersen M. Influence of shading control patterns on the energy assessment of office spaces, Energy and Buildings 50 (2012) 35-48.

[5] Li DHW. A review of daylight illuminance determinations and energy implications, Applied Energy 87 (2010) 2109-2118.

[6] Tagliabue LC, Buzzetti M, Arosio B. Energy saving through the sun: Analysis of visual comfort and energy consumption in office space, Energy Procedia 30 (2012) 693-703.

[7] Foucquier A, Robert S, Suard F, Stephan L, Jay A. State of the art in building modelling and energy performances prediction: A review, Renewable and Sustainable Energy Reviews 23 (2013) 272-288.

[8] Košir M, Krainer A, Dovjak M, Kristl Ž. Automatically controlled daylighting for visual and nonvisual effects, Lighting Research and Technology 43 (2001) 439-455.

[9] Trobec Lah M, Zupančič B, Peternelj J, Krainer A. Daylight illuminance control with fuzzy logic, Solar Energy 80 (2006) 307-321.

[10] Kristl Ž, Košir M, Trobec Lah M, Krainer A. Fuzzy control system for thermal and visual comfort in building, Renewable Energy 33 (2008) 694-702.

[11] Oh MH, Lee KH, Yoon JH. Automated control strategies of inside slat-type blind considering visual comfort and building energy performance, Energy and Buildings 55 (2012) 728-737. 
[12] Yun GY, Kim H, Kim JT. Effects of occupancy and lighting use patterns on lighting energy consumption, Energy and Buildings 46 (2012) 152-158.

[13] Košir M, Krainer A, Kristl Ž. Integral control system of indoor environment in continuously occupied spaces, Automation in Construction 21 (2012) 199-209.

[14] Li DHW, Lau CCS, Lam JC. Predicting daylight illuminance by computer simulation techniques, Lighting Research and Technology 36 (2004) 113-129.

[15] Li DHW, Cheung GHW, Lau CCS. A simplified procedure for determining indoor daylight illuminance using daylight coefficient concept, Building and Environment 41 (2006) 578-589.

[16] Kazanasmaz T, Günaydin M, Binol S. Artificial neural networks to predict daylight illuminance in office buildings, Building and Environment 44 (2009) 1751-1757.

[17] Lindelöf D. A fast daylight model suitable for embedded controllers, Solar Energy 83 (2009) 57-68.

[18] Larson GW, Shakespeare RA. Rendering with Radiance, Morgan Kaufmann Publishers, San Francisco, 2004.

[19] Reinhart C, Breton PF. Experimental validation of 3ds Max Design 2009 and Daysim 3.0, Eleventh International IBPSA Conference (2009), Glasgow, Scotland, 15141521.

[20] Laouadi A, Arsenault C. Validation of Skyvision, Institute of Research in Construction, Ottawa, 2004.
[21] Reinhart CF, Andersen M. Development and validation of a Radiance model for a translucent panel, Energy and Buildings 38 (2006) 890-904.

[22] Mardaljevic J. Validation of a lighting simulation program under real sky conditions, Lighting Research and Technology, 27 (1995) 181-188.

[23] Takagi T, Sugeno M. Fuzzy identification of systems and its applications to modelling and control, IEEE Transactions on Systems Man and Cybernetics 15 (1985) 116-132.

[24] Wang LX, Mendel JM. Fuzzy basis functions, universal approximation, and orthogonal least-squares learning, IEEE Transactions on Neural Networks 3 (1992) 807814.

[25] Kosko B. Fuzzy systems as universal approximators, IEEE Transactions on Computers 43 (1994) 1329-1333.

[26] Ying GH. Necessary conditions for some typical fuzzy systems as universal approximators, Automatica 33 (1997) 1333-1338.

[27] Golberg RR. Methods of real analysis, John Wiley and Sons, New York, 1976.

[28] Lin CH. Siso nonlinear system identification using a fuzzy-neural hybrid system, International Journal on Neural Systems 8 (1997) 325-337. 